\title{
EVALUATION OF UNCERTAINTY OF PHASE DIFFERENCE DETERMINATION IN PRESENCE OF BIAS
}

\author{
Lazar V. Saranovac1), Nada M. Vučijak ${ }^{2)}$ \\ 1) University of Belgrade, Faculty of Electrical Engineering, 11000 Belgrade, Serbia (laza@el.etf.rs) \\ 2) Elektrotehnički Institut DEC DOO, Kej 2. Oktobra 13,23000 Zrenjanin, Serbia \nadavucijak@gmail.com, +38164 2016010)
}

\begin{abstract}
Determination of the phase difference between two sinusoidal signals with noise components using samples of these signals is of interest in many measurement systems. The samples of signals are processed by one of many algorithms, such as 7PSF, UQDE and MSAL, to determine the phase difference. The phase difference result must be accompanied with estimation of the measurement uncertainty. The following issues are covered in this paper: the MSAL algorithm background, the ways of treating the bias influence on the phase difference result, comparison of results obtained by applying MSAL and the other mentioned algorithms to the same real signal samples, and evaluation of the uncertainty of the phase difference.
\end{abstract}

Keywords: sinusoidal electrical signals, phase difference, bias, uncertainty of measurement, MSAL algorithm.

(C) 2016 Polish Academy of Sciences. All rights reserved

\section{Introduction}

The phase difference between two sinusoidal signals is of interest in many measurement systems employed in such applications as: calibration of standards for electric power, determination of complex ratios between the output and input signals of transducers, voltage dividers and current shunts, flow measurement, source localization, etc. [1-9].

The phase difference between two continuous sinusoidal signals is usually determined by means of discrete samples of signals processed by one of many algorithms used for phase difference estimation. Some of the algorithms are: the three-parameter sinus fitting algorithm (3PSF), the four-parameter sinus fitting algorithm (4PSF), the seven-parameter sinus fitting algorithm (7PSF) [10-13], the unbiased quadrature delay estimator (UQDE) [9], and the modified simple algorithm (MSAL) [14]. According to the MSAL algorithm, two new signals' phases shifted to the existing signals are introduced, and a complex formula is applied to a large number of signal samples to determine the phase difference between two sinusoidal signals.

The result of phase difference measurement must be accompanied with estimation of the uncertainty according to the international recommendation GUM [15]. The measurement uncertainty is defined as a non-negative parameter characterizing the dispersion of quantity values being attributed to a measured value based on the information used [16]. The type A, type B, combined standard uncertainty and expanded uncertainty are defined in [15] and applied here. The measurement uncertainty can include components arising from systematic effects which are sometimes not corrected [15].

The rest of the paper is arranged as follows: Section 2 introduces the basic principles of MSAL algorithm. In Section 3 the bias of phase difference results determined from real signal samples is presented. Section 4 presents comparison of real signals' phase difference results obtained with the MSAL algorithm with those obtained with other algorithms. Section 5 introduces evaluation of the uncertainty of phase difference result. The uncertainty estimation 
results for samples of two voltages are presented and the uncertainty of phase difference result obtained by applying the MSAL algorithm to those samples is estimated. Section 6 presents the conclusions.

\section{MSAL algorithm}

Two real-valued sinusoidal signals without a dc offset can be expressed as:

$$
\begin{aligned}
& u_{1}(t)=U_{1} \sin \left(\omega_{0} t+\varphi_{1}\right)+n_{1}(t), \\
& u_{2}(t)=U_{2} \sin \left(\omega_{0} t+\varphi_{2}\right)+n_{2}(t),
\end{aligned}
$$

where: $U_{1}$ and $U_{2}$ are the amplitudes, $\omega_{0}$ is the angular frequency, $\varphi_{1}$ and $\varphi_{2}$ are the initial phases, $n_{1}(t)$ and $n_{2}(t)$ are the uncorrelated additive white Gaussian noise values [14]. The phase difference to be determined is $\varphi=\varphi_{1}-\varphi_{2}$.

To evaluate the phase difference $\varphi$, the phase-delayed signals in respect to (1) are introduced:

$$
\begin{aligned}
& u_{1}^{\prime}(t)=U_{1} \sin \left(\omega_{0} t+\varphi_{1}-\psi_{1}\right)+n_{1}^{\prime}(t), \\
& u_{2}^{\prime}(t)=U_{2} \sin \left(\omega_{0} t+\varphi_{2}-\psi_{2}\right)+n_{2}^{\prime}(t),
\end{aligned}
$$

where $\psi_{1}$ and $\psi_{2}$ are the known phase delays, and $n_{1}^{\prime}(t)$ and $n_{2}^{\prime}(t)$ are the phase-shifted noise values $n_{1}(t)$ and $n_{2}(t)$, respectively.

Using the signals defined by (1) and (2) the following combinations of signals are formed:

$$
\begin{aligned}
& m_{a}(t)=u_{1}(t) \cdot u_{2}(t)+u_{1}^{\prime}(t) \cdot u_{2}^{\prime}(t), \\
& m_{r}(t)=u_{1}^{\prime}(t) \cdot u_{2}(t)-u_{1}(t) \cdot u_{2}^{\prime}(t) .
\end{aligned}
$$

Substituting (1) and (2) in (3) gives the following results:

$$
\begin{aligned}
m_{a}(t) & =U_{1} U_{2} \cos \left(\varphi_{1}-\varphi_{2}+\frac{\psi_{2}-\psi_{1}}{2}\right) \cos \left(\frac{\psi_{2}-\psi_{1}}{2}\right) \\
& -U_{1} U_{2} \cos \left(2 \omega_{0} t+\varphi_{1}+\varphi_{2}+\frac{\psi_{1}+\psi_{2}}{2}\right) \cos \left(\frac{\psi_{1}+\psi_{2}}{2}\right), \\
& +n_{a}(t) \\
m_{r}(t) & =U_{1} U_{2} \sin \left(\varphi_{1}-\varphi_{2}+\frac{\psi_{2}-\psi_{1}}{2}\right) \sin \left(\frac{\psi_{1}+\psi_{2}}{2}\right) \\
& -U_{1} U_{2} \sin \left(2 \omega_{0} t+\varphi_{1}+\varphi_{2}+\frac{\psi_{1}+\psi_{2}}{2}\right) \sin \left(\frac{\psi_{2}-\psi_{1}}{2}\right), \\
& +n_{r}(t)
\end{aligned}
$$

where $n_{a}(t)$ and $n_{r}(t)$ contain all components with multiplicative noise. For uncorrelated white Gaussian noise the mean values of these components are zero.

For the sampled signals (1) and (2), with the sampling period $T_{s}$, the same results as given by (4) can be obtained for $m_{a}\left(k T_{s}\right)$ and $m_{r}\left(k T_{s}\right)$, computed at the instant $k T_{s}$, where $k$ is an integer. In a digital system, it is easy to achieve the same introduced phase delay for both 
introduced signals, i.e. $\psi_{1}=\psi_{2}=\psi$. For a discrete signal $u\left(k T_{s}\right)$, the phase delay can be obtained as a delay of its samples, so that $u^{\prime}\left(k T_{s}\right)=u\left(k T_{s}-r T_{s}\right)$, where $r$ is an integer.

When introduced, the phase delay is chosen to satisfy the condition:

$$
\psi_{1}=\psi_{2}=\psi=(4 l+1) \pi / 2,
$$

where $l$ is an integer. Next, the equations can be obtained:

$$
\begin{gathered}
m_{a}\left(k T_{s}\right)=U_{1} U_{2} \cos \left(\varphi_{1}-\varphi_{2}\right)+n_{a}\left(k T_{s}\right), \\
m_{r}\left(k T_{s}\right)=U_{1} U_{2} \sin \left(\varphi_{1}-\varphi_{2}\right)+n_{r}\left(k T_{s}\right) .
\end{gathered}
$$

For a high signal to noise ratio $(S N R)$, the phase difference $\varphi$ is obtained from (6):

$$
\varphi=\varphi_{1}-\varphi_{2} \approx \arctan \frac{m_{r}\left(k T_{s}\right)}{m_{a}\left(k T_{s}\right)},
$$

and this formula is called the SAL algorithm. It should be noticed that this formula for calculations needs only four samples of signals, one sample of each of four signals. is:

In the case of coherent sampling, the relation between signal and sampling angular frequency

$$
\omega_{0} / \omega_{s}=q / p
$$

where $q$ and $p$ are positive relative prime integers and $\omega_{s}=2 \pi / T_{s}$. According to (5) and (8), for the SAL algorithm, $r$ must be equal to:

$$
r=(4 l+1) p /(4 q) \text {. }
$$

When the equation (9) is not possible to satisfy, and/or $S N R$ is low, $M_{a}$ and $M_{r}$ are defined instead of $m_{a}\left(k T_{s}\right)$ and $m_{r}\left(k T_{s}\right)$ :

$$
\begin{gathered}
M_{a}=\frac{1}{n} \sum_{k=0}^{n-1} m_{a}\left(k T_{s}\right) \approx U_{1} U_{2} \cos \left(\varphi_{1}-\varphi_{2}\right), \\
M_{r}=\frac{1}{n} \sum_{k=0}^{n-1} m_{r}\left(k T_{s}\right) \approx \sin (\psi) U_{1} U_{2} \sin \left(\varphi_{1}-\varphi_{2}\right),
\end{gathered}
$$

where $n$ is the number of samples and should be an integer multiple of $p$, and $\psi \neq a \pi$, where $a$ is an integer. The introduced phase delay $\psi=r(q / p) 2 \pi$ may or may not satisfy (5). The estimated phase difference $\hat{\varphi}_{M S A L}$ can be calculated from (10) as [14]:

$$
\hat{\varphi}_{M S A L}=\arctan \frac{\sum_{k=0}^{n-1} m_{r}\left(k T_{s}\right)}{\sin \left(r \frac{q}{p} 2 \pi\right) \sum_{k=0}^{n-1} m_{a}\left(k T_{s}\right)} .
$$

This formula is called the modified simple algorithm (MSAL).

The detailed derivation of estimating the phase difference between two sinusoidal signals, as well as margins of errors for the SAL and MSAL algorithms in different situations is presented in [14]. The main advantage of these algorithms is reducing the computational complexity while maintaining good accuracy.

The values of phase difference determined using the MSAL algorithm applied to samples of two signals may contain a bias that comes from the measurement equipment and the fact that it 
is not possible to get a sampling frequency satisfying the requirement (8) of absolutely coherent sampling [17-18]. The consequences are: the requirement (5), i.e. the introduced phase delay of exactly $\pi / 2$, is not satisfied; the exact introduced phase delay is not known in (10).

The bias of an estimator is the difference between this estimator's expected value and the true value being estimated [19]. The measurement results can be corrected regarding the bias, but - since it is not required - an uncorrected bias can be included in the uncertainty statement [15].

\section{Bias of phase difference measurement results}

In the experimental measurement, two signals are generated by a phase standard, type 55002 Clarke Hess, with $50 \mathrm{~Hz}$ frequency and $10^{\circ}$ phase difference. For each of the two signals 200 samples are taken in one signal period, simultaneously using two type 3458A Agilent digital multimeters as sampling voltmeters (SV). These sampling voltmeters have a high-resolution integrating $\mathrm{ADC}$ (IADC) that operates according to the dual slope principle. The sampling process is such that the sampling instant is not a discrete point in time, but the measured signal is integrated by IADC over the aperture time of AD converter [20-27].

The results of phase difference between two real sinusoidal signals determined using the MSAL algorithm, are shown in Table 1. For $r=0$ and $r=100$ samples, or the introduced phase delay equal to $a \pi$, the algorithm is not defined. Theoretically, the introduced phase delay may be contained in an interval $(a \pi, a \pi+\pi)$, but in practice the introduced phase delay near $(2 l+1) \pi / 2$ is recommended because the error of phase difference result that comes from the function $\sin (x)$ in (11) is minimal [14]. In this example, the number of samples in a signal period is divisible by 4 , so that the introduced phase delay of approximately $\pi / 2$ can be obtained with 50 sample delays. The result for this case was adopted as the reference value.

In Table 1 the bias values for phase difference results determined using the MSAL algorithm are presented in dependence of the introduced phase delay, for $r$ samples. It can be seen that the bias has a value of $-64 \mathrm{~m}^{\circ}$ for $r=99$ samples, or near $\pi$. The bias is much smaller when the introduced phase delay is obtained for near 50 samples, i.e. $\pi / 2$.

Table 1. The bias value for phase difference between two sinusoidal signals determined using the MSAL algorithm for the phase delay ( $r$ samples) of introduced signals; an example with 200 samples in a period of each signal.

\begin{tabular}{|c|c|c|c|c|c|c|}
\hline$r$ (samples) & 1 & 10 & 15 & 30 & 40 & 50 \\
\hline$\varphi\left[^{\circ}\right]$ & 10.002 & 10.002 & 10.002 & 10.001 & 10.001 & 10.001 \\
\hline bias $\delta\left[\mathrm{m}^{\circ}\right]$ & 1 & 1 & 1 & 0 & 0 & REF \\
\hline$r$ (samples) & 65 & 75 & 85 & 97 & 98 & 99 \\
\hline$\varphi\left[^{\circ}\right]$ & 10.000 & 9.999 & 9.998 & 9.980 & 9.969 & 9.937 \\
\hline bias $\delta\left[\mathrm{m}^{\circ}\right]$ & -1 & -2 & -3 & -21 & -32 & -64 \\
\hline
\end{tabular}

\section{Comparison of results}

Next, there is presented the case of experimental measurement when the generated phase difference between two real sinusoidal signals with $53 \mathrm{~Hz}$ frequency is $59.9^{\circ}$. The samples are taken in 19 periods of each signal with 18 samples in a signal period. In contrast to the measurement in Section 3, the number of samples in a signal period is not divisible by 4 and because of that it is not possible to get $\pi / 2$ for the introduced phase delay when applying the UQDE and MSAL algorithms. 
The results of phase difference obtained by applying 7PSF, UQDE and MSAL to the same samples of two signals are shown in Table 2. MSAL4 denotes the MSAL algorithm when the introduced signals have the phase delay related to the original signals equal to 4 samples; MSAL5 denotes the MSAL algorithm when the introduced signals have the phase delay related to the original signals equal to 5 samples; UQDE4 and UQDE5 are denoted in an analogous way.

Table 2. The results of phase difference between two real sinusoidal signals determined by the 7PSF, UQDE4, UQDE5, MSAL4 and MSAL5 algorithms for chosen numbers of signal samples; an example with 18 samples in a period of each signal.

\begin{tabular}{|c|c|c|c|c|c|c|c|}
\cline { 2 - 8 } \multicolumn{2}{c|}{} & $\begin{array}{c}\text { 7PSF } \\
\text { algorithm }\end{array}$ & $\begin{array}{c}(\text { UQDE4 } \\
-7 P S F)\end{array}$ & $\begin{array}{c}\text { (UQDE5 } \\
-7 P S F)\end{array}$ & $\begin{array}{c}\text { (MSAL4 } \\
-7 P S F)\end{array}$ & $\begin{array}{c}\text { (MSAL5 } \\
-7 P S F)\end{array}$ & $\begin{array}{c}\text { MSALav } \\
-7 P S F\end{array}$ \\
\hline Samples & Periods & $\varphi\left[^{\circ}\right]$ & {$\left[\mathrm{m}^{\circ}\right]$} & {$\left[\mathrm{m}^{\circ}\right]$} & {$\left[\mathrm{m}^{\circ}\right]$} & {$\left[\mathrm{m}^{\circ}\right]$} & {$\left[\mathrm{m}^{\circ}\right]$} \\
\hline 18 & 1 & $\mathbf{5 9 . 9 0 0 0 9}$ & -381.11 & -380.99 & $\mathbf{0 . 9 0}$ & $\mathbf{1 . 0 1}$ & 0.95 \\
\hline 36 & 2 & $\mathbf{5 9 . 9 0 0 5 5}$ & -382.01 & -381.86 & $-\mathbf{0 . 0 0 4}$ & $\mathbf{0 . 1 5}$ & 0.07 \\
\hline 54 & 3 & $\mathbf{5 9 . 9 0 0 6 9}$ & -382.09 & -381.97 & $-\mathbf{0 . 0 9}$ & $\mathbf{0 . 0 4}$ & -0.02 \\
\hline 72 & 4 & $\mathbf{5 9 . 9 0 0 7 6}$ & -382.04 & -381.98 & $-\mathbf{0 . 0 3}$ & $\mathbf{0 . 0 3}$ & -0.002 \\
\hline 90 & 5 & $\mathbf{5 9 . 9 0 0 7 7}$ & -382.01 & -382.00 & $\mathbf{0 . 0 0 0 1}$ & $\mathbf{0 . 0 1}$ & 0.003 \\
\hline 108 & 6 & $\mathbf{5 9 . 9 0 0 6 9}$ & -381.99 & -381.99 & $\mathbf{0 . 0 2}$ & $\mathbf{0 . 0 2}$ & 0.02 \\
\hline 126 & 7 & $\mathbf{5 9 . 9 0 0 3 8}$ & -381.98 & -381.95 & $\mathbf{0 . 0 3}$ & $\mathbf{0 . 0 6}$ & 0.05 \\
\hline 144 & 8 & $\mathbf{5 9 . 9 0 0 1 0}$ & -382.00 & -381.97 & $\mathbf{0 . 0 1}$ & $\mathbf{0 . 0 4}$ & 0.03 \\
\hline 162 & 9 & $\mathbf{5 9 . 8 9 9 8 9}$ & -382.03 & -382.00 & $-\mathbf{0 . 0 1}$ & $\mathbf{0 . 0 2}$ & 0.001 \\
\hline 180 & 10 & $\mathbf{5 9 . 8 9 9 6 8}$ & -382.01 & -381.98 & $\mathbf{0 . 0 1}$ & $\mathbf{0 . 0 4}$ & 0.02 \\
\hline 198 & 11 & $\mathbf{5 9 . 8 9 9 6 0}$ & -381.98 & -381.97 & $\mathbf{0 . 0 3}$ & $\mathbf{0 . 0 4}$ & 0.04 \\
\hline 342 & 19 & $\mathbf{5 9 . 9 0 1 1 4}$ & -381.96 & -381.92 & $\mathbf{0 . 0 5}$ & $\mathbf{0 . 0 8}$ & 0.07 \\
\hline
\end{tabular}

The phase difference results obtained using the UQDE and MSAL algorithms are compared with those obtained using the 7PSF algorithm because it is shown in [13] that the 7PSF algorithm gives the most accurate results.

In Table 2: (UQDE4 - 7PSF) in millidegrees $\left[\mathrm{m}^{\circ}\right]$ denotes the difference between the phase difference results obtained using the UQDE4 and 7PSF algorithms; analogous meanings have: (UQDE5 - 7PSF), (MSAL4 - 7PSF), (MSAL5 - 7PSF), and (MSALav - 7PSF), where MSALav $=($ MSAL4+MSAL5 $) / 2$. The bias results obtained using all algorithms are compared with those obtained using 7PSF for estimation of phase difference. It can be seen in Table 2 that the minimum values are obtained in (MSAL4 - 7PSF) and (MSALav - 7PSF) columns. That means that the MSAL4 algorithm gives the phase difference results with almost the same accuracy as the 7PSF algorithm in the measurement points given in Table 2.

\section{Uncertainty of phase difference measurement}

Estimation of the uncertainty presented in this section concerns the phase difference measurement results obtained by applying the MSAL algorithm. The experimental measurement results from Section 4 are shown in dependence of the introduced phase delay in intervals: $(0, \pi)$ and $(\pi, 2 \pi)$.

\subsection{Estimation of uncertainty for samples of voltages}

The sinusoidal voltage, without a dc offset and noise, applied at the SV input is given by [28]: 


$$
u(t)=U \sin \left(\omega_{0} t\right)=U \sin \left(\frac{2 \pi}{T_{0}+\varepsilon_{J T o}} t\right),
$$

where $T_{0}$ is a signal period and $\varepsilon_{J T o}$ is a time jitter of the period $T_{0}$. The model of internal IADC of $\mathrm{SV}$ is given by the equation $[25,28]$ :

$$
U_{k}=\frac{1}{T_{i}+\varepsilon_{J T i}}\left(1+\varepsilon_{R E F}+\varepsilon_{G}+\frac{\varepsilon_{L I N}+\varepsilon_{R E S}}{A b s\left[U_{k}\right]} U_{F S}\right)^{\substack{k T_{s}+\varepsilon_{J S}+T_{i}+\varepsilon_{J T i} \\ k T_{s}+\varepsilon_{J T s}}} u(t) d t,
$$

where: $U_{k}$ is a sampled value at the instant $k T_{s} ; \varepsilon_{R E F}$ is the deviation of ideal internal DC reference of SV; $\varepsilon_{G}$ is the gain error of SV dependent on the integration time $T_{i} ; \varepsilon_{R E S}$ is an error due to SV resolution; $\varepsilon_{L I N}$ is an error due to linearity of SV; $U_{F S}$ is the voltage range of SV; $\varepsilon_{J T S}$ is the time jitter of the sampling period $T_{s} ; \varepsilon_{J T i}$ is the time jitter of the integration time $T_{i}$.

The standard uncertainty, type B [15], of each sample $U_{1 k}$ of the first voltage $u_{1}(t)$, taken by the first SV, denoted $u_{\mathrm{cB}}\left(U_{1 k}\right)$ is:

$$
\begin{aligned}
u_{\mathrm{cB}}\left(U_{1 k}\right)= & {\left[c_{1}^{2} u^{2}\left(\varepsilon_{R E F 1}\right)+c_{2}^{2} u^{2}\left(\varepsilon_{G 1}\right)+c_{3}^{2} u^{2}\left(\varepsilon_{R E S 1}\right)+c_{4}^{2} u^{2}\left(\varepsilon_{L I N 1}\right)+c_{5}^{2} u^{2}\left(\varepsilon_{J T o 1}\right),\right.} \\
& \left.+c_{6}^{2} u^{2}\left(\varepsilon_{J T S 1}\right)+c_{7}^{2} u^{2}\left(\varepsilon_{J T i 1}\right)+u_{w 1}^{2}\right]^{1 / 2}
\end{aligned}
$$

where: $c_{1}$ to $c_{7}$ are sensitivity coefficients for each sample $U_{1 k}, u\left(\varepsilon_{R E F 1}\right), u\left(\varepsilon_{G 1}\right), u\left(\varepsilon_{R E S 1}\right)$, $u\left(\varepsilon_{L I N 1}\right), u\left(\varepsilon_{J T O 1}\right), u\left(\varepsilon_{J T S 1}\right), u\left(\varepsilon_{J T i 1}\right)$ are standard uncertainties due to $\varepsilon_{R E F 1}, \varepsilon_{G 1}, \varepsilon_{R E S 1}, \varepsilon_{L I N 1}, \varepsilon_{J T S 1}$ $\varepsilon_{J T O 1}, \varepsilon_{J T T 1}$, respectively, and $u_{w 1}^{2}$ is the variance due to noise from the signal source and sampling the first signal. Some values of the uncertainties in (14) are derived from the manufacturer specifications of SV, whereas others - from the calibration certificate of SV.

The sensitivity coefficients [15] for each sample $U_{1 k}$ are calculated using (12) and (13) according to:

$$
\begin{gathered}
c_{1}=\frac{\partial U_{1 k}}{\partial \varepsilon_{R E F 1}}, c_{2}=\frac{\partial U_{1 k}}{\partial \varepsilon_{G 1}}, c_{3}=\frac{\partial U_{1 k}}{\partial \varepsilon_{R E S 1}}, c_{4}=\frac{\partial U_{1 k}}{\partial \varepsilon_{L I N 1}}, \\
c_{5}=\frac{\partial U_{1 k}}{\partial \varepsilon_{J T o 1}}, c_{6}=\frac{\partial U_{1 k}}{\partial \varepsilon_{J T s 1}}, c_{7}=\frac{\partial U_{1 k}}{\partial \varepsilon_{J T i 1}} .
\end{gathered}
$$

The standard uncertainty, type $\mathrm{B}$, of each sample $U_{2 k}$ of the second voltage $u_{2}(t)$, taken by the second $\mathrm{SV}$, denoted $u_{\mathrm{cB}}\left(U_{2 k}\right)$, is given in an analogous way to (14):

$$
\begin{aligned}
u_{\mathrm{cB}}\left(U_{2 k}\right)= & {\left[c_{8}^{2} u^{2}\left(\varepsilon_{R E F 2}\right)+c_{9}^{2} u^{2}\left(\varepsilon_{G 2}\right)+c_{10}^{2} u^{2}\left(\varepsilon_{R E S 2}\right)+c_{11}^{2} u^{2}\left(\varepsilon_{L I N 2}\right)+c_{12}^{2} u^{2}\left(\varepsilon_{J T o 2}\right)\right.} \\
& \left.+c_{13}^{2} u^{2}\left(\varepsilon_{J T s 2}\right)+c_{14}^{2} u^{2}\left(\varepsilon_{J T i 2}\right)+u_{w 2}^{2}\right]^{1 / 2},
\end{aligned}
$$

where: $c_{8}$ to $c_{14}$ are sensitivity coefficients for each sample $U_{2 k}$ and standard uncertainties have analogous explanations to those in (14). The sensitivity coefficients are calculated in an analogous way to (15).

The standard uncertainty type A of each sample is calculated from 19 periods of signal according to GUM [15]. The combined standard uncertainties $u_{\mathrm{c}}\left(U_{1 k}\right)$ and $u_{\mathrm{c}}\left(U_{2 k}\right)$ for each of 18 samples in a period of two signals, are calculated from the type A and type B uncertainties, and are shown in Table 3. 
Table 3. The voltage samples $U_{1 k}$ and $U_{2 k}$ of two sinusoidal signals and the combined standard uncertainties $u_{\mathrm{c}}\left(U_{1 k}\right)$ and $u_{\mathrm{c}}\left(U_{2 k}\right)$ for an example with 18 samples in a period of each signal.

\begin{tabular}{|c|c|c|c|c|}
\hline \multirow{2}{*}{$k$} & $U_{1 k}$ & $u_{\mathrm{c}}\left(U_{1 k}\right)$ & $U_{2 k}$ & $u_{\mathrm{c}}\left(U_{2 k}\right)$ \\
\cline { 2 - 5 } & {$[\mathrm{V}]$} & {$[\mathrm{mV}]$} & {$[\mathrm{V}]$} & {$[\mathrm{mV}]$} \\
\hline 0 & 0.129951 & 0.1785 & 1.031419 & 0.0935 \\
\hline 1 & 0.504598 & 0.1554 & 1.122462 & 0.0717 \\
\hline 2 & 0.818463 & 0.1286 & 1.078107 & 0.0776 \\
\hline 3 & 1.033572 & 0.0922 & 0.903626 & 0.1070 \\
\hline 4 & 1.124001 & 0.0703 & 0.620167 & 0.1362 \\
\hline 5 & 1.078850 & 0.0796 & 0.262030 & 0.1555 \\
\hline 6 & 0.903546 & 0.1098 & -0.127721 & 0.1601 \\
\hline 7 & 0.619273 & 0.1399 & -0.502052 & 0.1493 \\
\hline 8 & 0.260405 & 0.1584 & -0.815880 & 0.1245 \\
\hline 9 & -0.129899 & 0.1622 & -1.031253 & 0.0938 \\
\hline 10 & -0.504526 & 0.1498 & -1.122210 & 0.0716 \\
\hline 11 & -0.818356 & 0.1248 & -1.077772 & 0.0793 \\
\hline 12 & -1.033465 & 0.0927 & -0.903321 & 0.1076 \\
\hline 13 & -1.123912 & 0.0704 & -0.619898 & 0.1364 \\
\hline 14 & -1.078824 & 0.0793 & -0.261827 & 0.1549 \\
\hline 15 & -0.903589 & 0.1097 & 0.127784 & 0.1586 \\
\hline 16 & -0.619357 & 0.1398 & 0.502035 & 0.1479 \\
\hline 17 & -0.260512 & 0.1605 & 0.815872 & 0.1186 \\
\hline
\end{tabular}

\subsection{Uncertainty of phase difference result obtained by MSAL algorithm}

The equation (11) can be written as:

$$
\hat{\varphi}_{M S A L}=\arctan \frac{\sum_{k=0}^{n-1}\left(M_{1 k}-M_{3 k}\right)}{\sin \left(r \frac{q}{p} 2 \pi\right) \sum_{k=0}^{n-1}\left(M_{2 k}+M_{4 k}\right)}=\arctan \frac{M_{1}-M_{3}}{\sin \left(r \frac{q}{p} 2 \pi\right)\left(M_{2}+M_{4}\right)},
$$

where:

$$
M_{1 k}=U_{1 k}^{\prime} U_{2 k}, M_{3 k}=U_{1 k} U_{2 k}^{\prime}, M_{3 k}=U_{1 k} U_{2 k}^{\prime}, M_{4 k}=U_{1 k}^{\prime} U_{2 k}^{\prime},
$$

$U_{1 k}^{\prime}$ are phase delayed samples $U_{1 k}, U_{2 k}^{\prime}$ are phase delayed samples $U_{2 k}$ and

$$
M_{1}=\sum_{k=0}^{n-1} M_{1 k}, M_{2}=\sum_{k=0}^{n-1} M_{2 k}, M_{3}=\sum_{k=0}^{n-1} M_{3 k}, M_{4}=\sum_{k=0}^{n-1} M_{4 k}
$$

The combined standard uncertainty for the phase difference $u_{\mathrm{c}}\left(\hat{\varphi}_{M S A L}\right)$ obtained using the MSAL algorithm, according to GUM [15], is:

$$
\begin{aligned}
u_{\mathrm{c}}\left(\hat{\varphi}_{M S A L}\right)= & {\left[c_{15}^{2} u^{2}\left(M_{1}\right)+c_{16}^{2} u^{2}\left(M_{2}\right)+c_{17}^{2} u^{2}\left(M_{3}\right)+c_{18}^{2} u^{2}\left(M_{4}\right)\right.} \\
& +u_{\mathrm{cA}}^{2}(\varphi)+2 c_{15} c_{16} u\left(M_{1}\right) u\left(M_{2}\right) r_{\mathrm{C}}\left(M_{1}, M_{2}\right)+2 c_{15} c_{17} u\left(M_{1}\right) u\left(M_{3}\right) r_{\mathrm{C}}\left(M_{1}, M_{3}\right), \\
& +2 c_{15} c_{18} u\left(M_{1}\right) u\left(M_{4}\right) r_{\mathrm{C}}\left(M_{1}, M_{4}\right)+2 c_{16} c_{17} u\left(M_{2}\right) u\left(M_{3}\right) r_{\mathrm{C}}\left(M_{2}, M_{3}\right) \\
& \left.+2 c_{16} c_{18} u\left(M_{2}\right) u\left(M_{4}\right) r_{\mathrm{C}}\left(M_{2}, M_{4}\right)+2 c_{17} c_{18} u\left(M_{3}\right) u\left(M_{4}\right) r_{\mathrm{C}}\left(M_{3}, M_{4}\right)\right]^{1 / 2}
\end{aligned}
$$

where $u_{\mathrm{cA}}(\varphi)$ is the standard uncertainty type $\mathrm{A}$, the correlation coefficient is $r_{\mathrm{C}}$ and sensitivity coefficients are calculated from the formulas: 


$$
c_{15}=\frac{\partial \hat{\varphi}_{M S A L}}{\partial M_{1}}, c_{16}=\frac{\partial \hat{\varphi}_{M S A L}}{\partial M_{2}}, c_{17}=\frac{\partial \hat{\varphi}_{M S A L}}{\partial M_{3}}, c_{18}=\frac{\partial \hat{\varphi}_{M S A L}}{\partial M_{4}} .
$$

The standard uncertainty type A for the phase difference $u_{\mathrm{cA}}(\varphi)$ is determined from the phase difference values calculated for each of 19 periods of signals using the MSAL algorithm. From those 19 values, the standard deviation and standard uncertainty type A are calculated according to GUM.

The square of standard uncertainty $u^{2}\left(M_{1}\right)$ in (20) can be written as:

$$
u^{2}\left(M_{1}\right)=u^{2}\left(\sum_{k=0}^{n-1} M_{1 k}\right)=\sum_{k=0}^{n-1} u^{2}\left(M_{1 k}\right) .
$$

In an analogous way the squares of standard uncertainty components $u^{2}\left(M_{2}\right), u^{2}\left(M_{3}\right)$ and $u^{2}\left(M_{4}\right)$ in (20) can be written.

The square of standard uncertainty of $M_{1 k}$ is given by [15]:

$$
\begin{aligned}
u^{2}\left(M_{1 k}\right) & =\left(\frac{\partial M_{1 k}}{\partial U_{1 k}^{\prime}}\right)^{2} u^{2}\left(U_{1 k}^{\prime}\right)+\left(\frac{\partial M_{1 k}}{\partial U_{2 k}}\right)^{2} u^{2}\left(U_{2 k}\right) \\
& +2\left(\frac{\partial M_{1 k}}{\partial U_{1 k}^{\prime}}\right)\left(\frac{\partial M_{1 k}}{\partial U_{2 k}}\right) u\left(U_{1 k}^{\prime}\right) u\left(U_{2 k}\right) r_{\mathrm{C}}\left(U_{1 k}^{\prime}, U_{2 k}\right) \\
& =U_{2 k}^{2} u^{2}\left(U_{1 k}^{\prime}\right)+U_{1 k}^{\prime 2} u^{2}\left(U_{2 k}\right)+2 U_{2 k} U_{1 k}^{\prime} u\left(U_{1 k}^{\prime}\right) u\left(U_{2 k}\right) r_{\mathrm{C}}\left(U_{1 k}^{\prime}, U_{2 k}\right),
\end{aligned}
$$

where $r_{\mathrm{C}}\left(U_{1 k}^{\prime}, U_{2 k}\right)$ is the correlation coefficient between samples of two voltages: $U_{1 k}^{\prime}$ and $U_{2 k}$.

The squares of standard uncertainties: $u^{2}\left(M_{2 k}\right), u^{2}\left(M_{3 k}\right), u^{2}\left(M_{4 k}\right)$ can be obtained in an analogous way.

In the measurement system two SVs are used, whose bandwidths are not exactly known and - according to the manufacturer's specification, for a chosen measurement range - the instrument-to-instrument difference of the upper limit of bandwidth is estimated to be in the range between $140 \mathrm{kHz}$ and $160 \mathrm{kHz}$. Simultaneous measurement of two voltages whose frequency is $53 \mathrm{~Hz}$ produces the phase difference error of $0.00271^{\circ}$, due to the influence of bandwidth [1]. Assuming a uniform distribution of possible values [15], the type B component of uncertainty in this case increases to $0.00157^{\circ}$.

The differences in delay time between EXT TRIG input channels of two SVs according to the manufacturer's specification may be up to $75 \mathrm{~ns}$, and that can produce the phase angle error whose value is $0.00143^{\circ}$ for signals with frequency $53 \mathrm{~Hz}$ [1]. Assuming a uniform distribution of possible values [15], the type B component of uncertainty in this case increases to $0.00083^{\circ}$.

The bias of results is a systematic error that can be applied as a correction of the measured values, but that is not essential and the bias can be expressed as a part of expanded uncertainty (GUM, F.2.4.5). Three methods are proposed for treating the uncorrected bias $\delta$ as a part of expanded uncertainty [29]:

1. „RSS $u_{\mathrm{c}}$ method“ treats the uncorrected bias $\delta$ as another uncertainty source and sums it in an RSS (root-sum-of-squares) way with the combined standard uncertainty $u_{\mathrm{c}}$ :

$$
U_{\mathrm{RSS} u_{\mathrm{c}}}=k \sqrt{u_{\mathrm{c}}^{2}+\delta^{2}}
$$

2. „RSSU method“ sums the bias $\delta$ in an RSS way with the expanded uncertainty $k u_{\mathrm{c}}$ :

$$
U_{\mathrm{RSS} U}=\sqrt{k^{2} u_{\mathrm{c}}^{2}+\delta^{2}}
$$


3. „SUMU method“" algebraically sums the bias $\delta$ with the expanded uncertainty $k u_{c}$ :

$$
U_{+}=\left\{\begin{array}{l}
k u_{\mathrm{c}}-\delta, \text { if } k u_{\mathrm{c}}-\delta>0 \\
0, \text { if } k u_{\mathrm{c}}-\delta \leq 0
\end{array} \text { and } U_{-}=\left\{\begin{array}{l}
k u_{\mathrm{c}}+\delta, \text { if } k u_{\mathrm{c}}+\delta>0 \\
0, \text { if } k u_{\mathrm{c}}+\delta \leq 0 .
\end{array}\right.\right.
$$

In (24) to (26) $u_{\mathrm{c}}$ is the combined standard uncertainty, $\delta$ denotes the bias, $k$ is a coverage factor, and $k u_{\mathrm{c}}$ is the expanded uncertainty.

The results for a phase difference of $59.9^{\circ}$ between two sinusoidal signals determined using the MSAL algorithm applied to 19 periods of signals, together with the expanded uncertainties, are shown in Figs. 1-3.

It can be seen:

a) for „RSS $u_{c}$ method“ the expanded uncertainty is:

$$
\text { from } \pm 4.2 \mathrm{~m}^{\circ} \text { (for } r=4 \text { ) to } \pm 14.9 \mathrm{~m}^{\circ} \text { (for } r=17 \text { ); }
$$

b) for „RSS $U$ method“ the expanded uncertainty is:

$$
\text { from } \pm 3.9 \mathrm{~m}^{\circ} \text { (for } r=1 \text { ) to } \pm 8.2 \mathrm{~m}^{\circ} \text { (for } r=17 \text { ); }
$$

c) for „SUM $U$ method" the expanded uncertainties are:

$$
\begin{aligned}
& \text { for } r=4, U_{+}=4.4 \mathrm{~m}^{\circ} \text { and } U_{-}=4.0 \mathrm{~m}^{\circ} ; \\
& \text { for } r=8, U_{+}=0.8 \mathrm{~m}^{\circ} \text { and } U_{-}=6.8 \mathrm{~m}^{\circ} ; \\
& \text { for } r=14, U_{+}=3.8 \mathrm{~m}^{\circ} \text { and } U_{-}=4.6 \mathrm{~m}^{\circ} ; \\
& \text { for } r=17, U_{+}=0.0 \mathrm{~m}^{\circ} \text { and } U_{-}=11.0 \mathrm{~m}^{\circ} .
\end{aligned}
$$

The most realistic case, shown in Fig. 3, is obtained with application of „SUMU method“. The same conclusion is presented in [29].

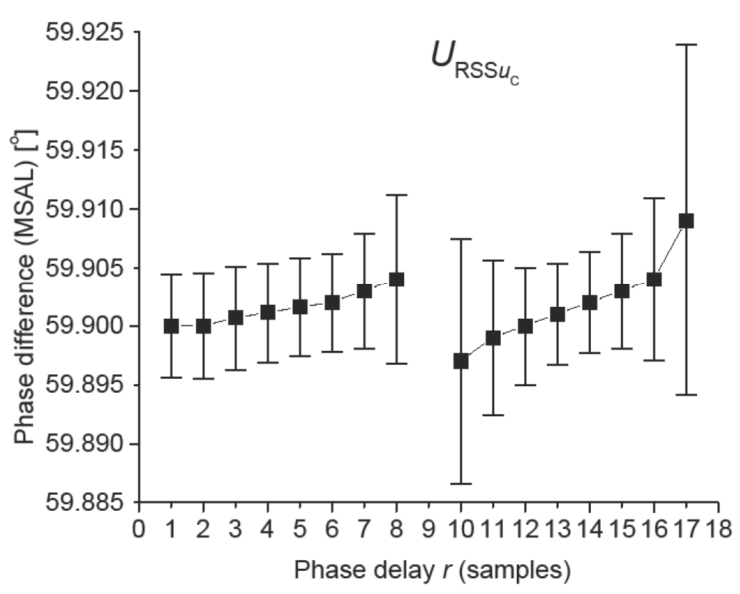

Fig. 1. The RSS $u_{\mathrm{c}}$ method for treating the uncorrected bias in evaluation of the uncertainty of the phase difference $59.9^{\circ}$ between two real sinusoidal signals determined using the MSAL algorithm. 


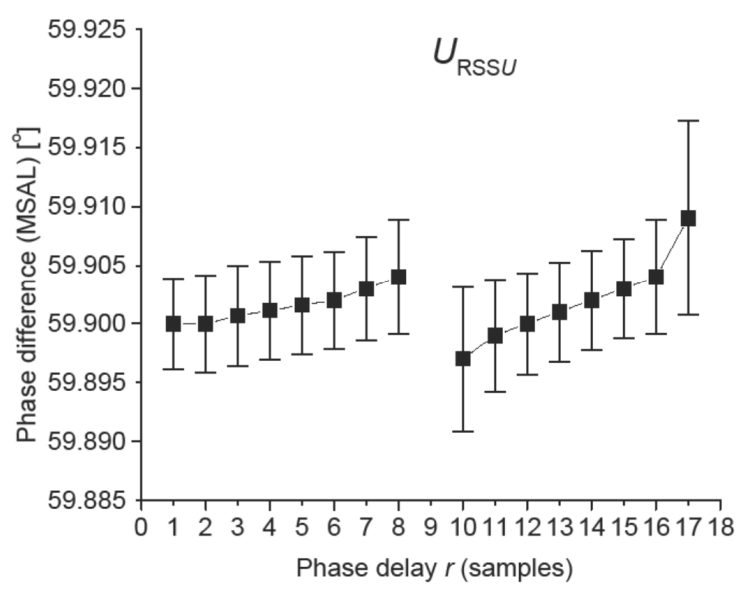

Fig. 2. The RSS $U$ method for treating the uncorrected bias in evaluation of the uncertainty of the phase difference $59.9^{\circ}$ between two real sinusoidal signals determined using the MSAL algorithm.

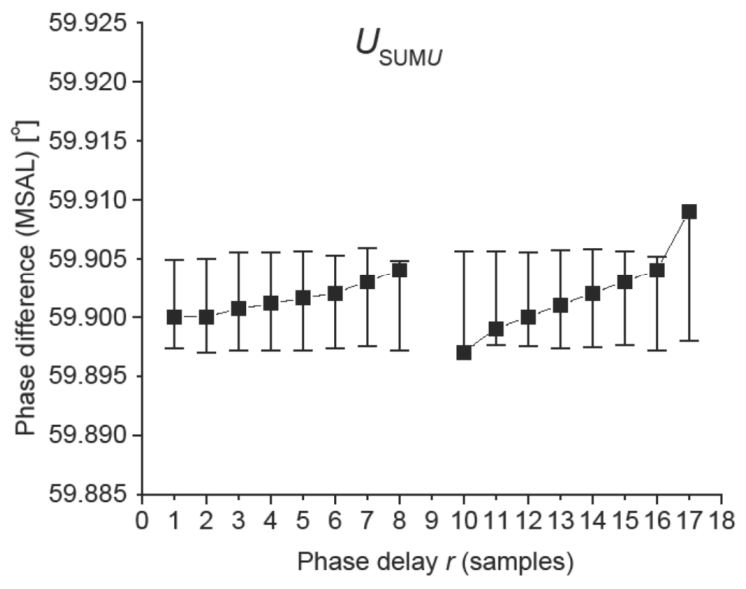

Fig. 3. The SUM $U$ method for treating the uncorrected bias in evaluation of the uncertainty of the phase difference $59.9^{\circ}$ between two real sinusoidal signals determined using the MSAL algorithm.

\section{Conclusions}

The phase difference between two sinusoidal signals is usually determined by using samples of signals processed by an algorithm estimating the phase difference. This paper presents application of the MSAL algorithm for determination of the phase difference and comparison of results with the ones obtained by using the 7PSF and UQDE algorithms. The differences between the phase difference results obtained by the MSAL algorithm, for which frequency is not known and those obtained by the 7PSF algorithm, for which frequency is known, are equal to parts of millidegrees.

The uncertainty of phase difference between two sinusoidal signals determined using the MSAL algorithm is estimated according to GUM. The bias of results is not applied as 
a correction of the measured values, but it is expressed - using three methods - as a part of the expanded uncertainty. The conclusion is that the most realistic in this case is the „SUMU method" which algebraically sums the bias with the expanded uncertainty.

\section{Acknowledgement}

The authors would like to thank the reviewers for their helpful comments and suggestions which improved the quality of this paper.

\section{References}

[1] Svensson, S. (1999). Power measurement techniques for non-sinusoidal conditions. PhD thesis, Chalmers university of technology, Goteborg, Sweden.

[2] Rydler, K.E., Svensson, S., Tarasso, V. (2002). Voltage dividers with low phase angle errors for a wideband power measuring system. IEEE CPEM 2002, Conference Digest, 382-383.

[3] Voljč, B., Lindič, M., Pinter B., Kokalj, M., Svetik, Z., Lapuh, R. (2012). New design of coaxial current shunts for 50 A and 100 A. IEEE CPEM 2012, Conference Digest, 222-223.

[4] Mohns, E., Ramm, G., Kürten Ihlenfeld, W.G., Palafox, L., Moser, H. (2009). The PTB Primary Standard for Electrical AC Power. MAPAN - Journal of Metrology Society of India, 24(1), 15-19.

[5] Kokalj, M., Pinter, B., Lindič, M., Ziade, F. (2014). Development of coaxial adapter for calibration of EMC devices. IEEE CPEM 2014, Conference Digest, 586-587.

[6] Svensson, S., Rydler, K.E., Tarasso, V. (2004). Improved model and phase-angle verification of current shunts for AC and power measurements. IEEE CPEM 2004, Conference Digest, 82-83.

[7] Kokalj, M., Lindič, M., Voljč, B., Pinter, B., Svetik, Z., Lapuh, R. (2012). High accuracy signal parameter estimation algorithm for calibration of PMU devices. IEEE CPEM 2012, Conference Digest, 288-289.

[8] Tu, Y., Yang, H., Zhang, H., Liu, X. (2014). CMF Signal Processing Method Based on Feedback Corrected ANF and Hilbert Transformation. Measurement Science Review, 14 (1), 41-47.

[9] So, H.C. (2005). A Comparative Study of Two Discrete - Time Phase Delay Estimators. IEEE Trans. Instrum. Meas., 54(6), 2501-2504.

[10] IEEE Standard 1241. (2010). Terminology and test methods for analog-to-digital converters.

[11] Ramos, P.M., Janeiro, F.M., Radil, T. (2010). Comparative analysis of three algorithms for two channel common frequency sinewave parameter estimation: ellipse fit, seven parameter sine fit and spectral sinc fit. Metrol. Meas. Syst., 17(2), 250-270.

[12] Vučijak, N., Radojević, N. (2005). Three, Four and Seven Parameters Sine-fitting Algorithms Applied in Electric Power Calibrations. EUROCON 2005, Computer as a tool, 2, 1148-1150.

[13] Sedlaček, M., Krumpholc, M. (2005). Digital measurement of phase difference - a comparative study of DSP algorithms. Metrol. Meas. Syst., 12(4), 427-448.

[14] Vučijak, N.M., Saranovac, L.V. (2010). A Simple Algorithm for Estimation of Phase Difference between Two Sinusoidal Voltages. IEEE Trans. Instrum. Meas., 59(12), 3152-3158.

[15] JCGM 100:2008 GUM 1995 with minor corrections „Evaluation of measurement data - Guide to the expression of uncertainty in measurement", Joint Committee for Guides in Metrology.

[16] JCGM 200:2008 International Vocabulary of Metrology - Basic and General Concepts and Associated Terms (VIM), BIPM.

[17] Hegeduš, H., Mostarac, P., Malarić, R. (2011). Comparison of RMS Value Measurement Algorithms of Noncoherent Sampled Signals. Measurement Science Review, 11(3), 79-84.

[18] Novotny, M., Sedlacek, M. (2004). Measurement of RMS values of non-coherently sampled signals. Proc. of the 13th International Symposium on Measurements for Research and Industry Applications, IMEKO TC4. Athens, Greece, 230-235. 
[19] Kay, S.M. (1993). Fundamentals of Statistical Signal Processing-Estimation Theory. Englewood Clifs, NJ: Prentice-Hall.

[20] Pogliano, U. (2001). Use of Integrative Analog-to-Digital Converters for High-Precision Measurement of Electrical Power. IEEE Trans. Instrum. Meas., 50(5), 1315-1318.

[21] Çayci, H. (2011). Final report on key comparison EURAMET.EM-K5.1 (EURAMET Project No. 687): Comparison of 50/60 Hz power. Metrologia 48, Technical supplement 01009.

[22] Di Lillo, L., Laiz, H., Yasuda, E., Garcia, R. (2009). Sampling wattmeter at INTI. VIII Semetro, Brazil.

[23] Mohns, E., Ramm, G., Kürten Ihlenfeld, W.G., Palafox, L., Moser, H. (2009). The PTB Primary Standard for Electrical AC Power. MAPAN - Journal of Metrology Society of India. 24(1), 15-19.

[24] Tóth, E., Franco, A.M.R., Debatin, R.M. (2005). Power and Energy Reference System, Applying DualChannel Sampling. IEEE Trans. Instrum. Meas., 54(1), 404-408.

[25] Kürten Ihlenfeld, W.G., Mohns, E., Bachmair, H., Ramm, G., Moser, H. (2003). Evaluation of the Synchronous Generation and Sampling Technique. IEEE Trans. Instrum. Meas., 52(2), 371-374.

[26] Espel, P., Poletaeff, A., Bounouh, A. (2009). Characterisation of analogue-to-digital converters of a commercial digital voltmeter in the $20 \mathrm{~Hz}$ to $400 \mathrm{~Hz}$ frequency range. Metrologia, 46, 578-584.

[27] Lapuh, R., Voljč, B., Lindič, M. (2015). Evaluation of Agilent 3458A Time Jitter Performance, Two Sampling Voltmeters. IEEE Trans. Instrum. Meas., 64(6), 1331-1335.

[28] Kürten Ihlenfeld, W.G., Mohns, E. (2004). AC-dc transfer measurement of highest accuracy with synchronous analogue-to-digital conversion. Metrologia, 41, 111-115.

[29] Phillips, S., Eberhardt, K. (1997). Guidelines for Expressing the Uncertainty of Measurement Results Containing Uncorrected Bias. Journal of Research of the National Institute of Standards and Technology, $102,577-585$. 